\title{
Marija Knezevic-Pogancev Specific features of migraine syndrome in children
}

Received: 16 April 2006

Accepted in revised form: 27 June 2006

Published online: 11 August 2006
M. Knezevic-Pogancev ( $ه$ ) Institute of Child and Youth Health Care, Novi Sad, Hajduk Veljkova 10, 21000 Novi Sad, Serbia \& Montenegro e-mail: godipo@eunet.yu

Tel.: 381-637789285

Fax: 381-21530543

\author{
Abstract The aim of the study was \\ to define factors that can be used to \\ distinguish migraine headaches \\ from primary non-migraine \\ headaches. Specific characteristics \\ of headaches were analysed in \\ 30636 children aged 3-17; 18.97\% \\ had recurrent primary non-migraine \\ headaches, whereas $8.63 \%$ had \\ migraine headaches. Migraine \\ attacks follow identical patterns \\ (94.9\%): occurring monthly \\ (78.0\%), occurring in morning \\ hours $(58.5 \%)$, lasting for several \\ hours $(45.1 \%)$ and ending after \\ sleep $(76.7 \%)$. Nausea, vomiting
}

impulse and vomiting are basic present elements of migraine attacks in children. Canonical discriminate analysis defined the following statistically significant factors, which can distinguish migraine headaches from primary non-migraine headaches in children: relief after sleep (0.945), vomiting impulse (0.945), photophobia (0.523), nausea (0.379), phonophobia $(0.354)$ and vomiting (0.330).

Key words Migraine $\cdot$ Signs and symptoms $\cdot$ Headache $\cdot$ Children

\section{Introduction}

Diagnosing migraine syndrome (MS) in children represents an everyday clinical problem. Different criteria are suggested as diagnostic of MS in children and adolescents $[1,2]$. Sensory, vegetative and affective phenomena, with marked quantitative and qualitative variations among children outline MS [3, 4]. MS clinical features are age-dependent [5]. The younger the child is, the harder it is to diagnose MS [6, 7]. There are no definite reliable principles for safe, accurate and fast diagnosis of MS in children.

This article aims to define those features that would provide easier and more precise diagnosis of MS in children.

\section{Material and methods}

The research was carried out in the territory of the northern province of Serbia, Vojvodina, which has a population of 2031992 people according to the last census (2002). From 1988 to 2004 participants were selected by a multistage stratified clustered sampling procedure from 23 preschools and 42 grade schools in 9 cities in Vojvodina (Novi Sad, Subotica, Kikinda, Zrenjanin, Vrsac, Bela Crkva, Melenci, Futog and Temerin). 30636 children aged 3-17 years (mean 9.21, SD 3.16) were surveyed (15 202 girls and 15434 boys).

A semi-structured questionnaire, developed by the Author, was designed according to the International Headache Society (IHS) criteria. The accuracy of the questionnaire used in this survey was based on the Classification and diagnostic criteria for headache disorders, cranial neuralgias and facial pain and 
International Headache Society-Revised (IHS-R) criteria [8, 9]. We acknowledged all migraine attack characteristics according to the second edition of the International Classification of Headache Disorders. The study was conducted in two phases: completing the questionnaire, and face-to-face interview for those having recurrent headaches. Based on data gathered through the questionnaire, possible migraine sufferers underwent an extended interview and neurological examination.

Canonical discrimination analysis and multiple regression correlation analysis were used to define specific characteristics that can distinguish migraine headaches from primary nonmigraine headaches.

\section{Results}

Out of 30636 observed children, 5812 (18.97\%) reported primary non-migraine recurrent headaches (2910 male and 2902 female) and $2644(8.63 \%)$ reported migraine headaches (1162 male and 1482 female). Migraine headache is reported for the first time in children aged 5.14 years (range 2.8-14 years). Migraine attacks follow identical patterns in 2509 (94.9\%) children: occurring monthly in $2062(78.0 \%)$, occurring in the morning hours in 1547 (58.5\%), lasting for several hours in $1192(45.1 \%)$ and ending after sleep in $2028(76.7 \%)$ children.

Migraine-associated photophobia is reported in 2372 (89.7\%) children, phonophobia in 2107 (79.7\%) children, and other vegetative symptoms (changes in skin colour, sweating, irregular breathing, precordial pain, etc.) in 2610 (98.7\%) children. Phonophobia is the dominant symptom in children at average age 11.11 years (range 3-17), phonophobia in children at average age of 11.21 years (range 3-17) and other vegetative symptoms in children aged 9.10 years (range 3-17). Nausea is present in 2607 (98.6\%), impulse to vomit in 2583 (97.7\%) and vomiting in $2583(77.2 \%)$ children with MS. Nausea, impulse to vomit and vomiting (all together) are dominant in children aged 11.10 years (range 3-17), presenting in 2607 (98.6\%) of all children. Recurrent abdominal pain with headache attacks is reported by 1966 (74.4\%) children with MS, mostly at age 7.3 years (range 3-17) (Table 1).

Canonical discriminate analysis pointed to the following statistically significant factors (canonical dis-

Table 2 Migraine headache-associated symptoms. Canonical discriminant analysis

\begin{tabular}{lr}
\hline Migraine symptom & Migraine and other headaches \\
\hline Vomiting instinct & 0.945 \\
Relief after sleep & 0.945 \\
Photophobia & 0.523 \\
Nausea & 0.379 \\
Phonophobia & 0.354 \\
Vomiting & 0.330 \\
Vertigo & 0.165 \\
Other & 0.057 \\
Abdominal pain & 0.051 \\
\hline
\end{tabular}

Table 1 Age-dependent symptoms in migraine headache

\begin{tabular}{|c|c|c|c|c|c|c|}
\hline Symptoms & $\begin{array}{l}\text { Headache/ } \\
\text { symptoms } \\
\text { presence }\end{array}$ & $\begin{array}{r}\text { Children with } \\
\text { migraine }\end{array}$ & $\begin{array}{r}\text { Arithmetical } \\
\text { mean }\end{array}$ & $\begin{array}{r}\text { Standard } \\
\text { deviation }\end{array}$ & $\begin{array}{r}\text { Pearson's } \\
\text { coefficient } \\
\text { of correlation }\end{array}$ & $\begin{array}{r}\text { Statistical significance } \\
\text { of correlation } \\
\text { coefficient }\end{array}$ \\
\hline \multirow[t]{3}{*}{ Phonophobia } & Minimal & 1081 & 11.21 & 2.90 & 0.09 & - \\
\hline & Maximal & 1563 & 11.00 & 3.16 & 0.1 & \\
\hline & Medium & 2644 & 11.11 & 3.04 & 0.07 & \\
\hline \multirow[t]{3}{*}{ Photophobia } & Minimal & 1166 & 11.34 & 2.80 & 0.08 & - \\
\hline & Maximal & 1478 & 11.12 & 3.16 & 0.1 & \\
\hline & Medium & 2644 & 11.21 & 3.06 & 0.05 & \\
\hline \multirow{3}{*}{$\begin{array}{l}\text { Other vegetative } \\
\text { symptoms }\end{array}$} & Minimal & 975 & 11.73 & 2.93 & 0.11 & 0.01 \\
\hline & Maximal & 1769 & 10.76 & 3.04 & 0.08 & \\
\hline & Medium & 2644 & 11.10 & 3.04 & 0.07 & \\
\hline \multirow{3}{*}{$\begin{array}{l}\text { Nausea, impulse } \\
\text { to vomit, vomiting }\end{array}$} & Minimal & 297 & 6.48 & 1.31 & 0.25 & 0.01 \\
\hline & Maximal & 2347 & 10.17 & 3.00 & 0.07 & \\
\hline & Medium & 2644 & 11.10 & 3.04 & 0.07 & \\
\hline \multirow[t]{3}{*}{ Abdominal pain } & Minimal & 678 & 12.27 & 2.15 & 0.06 & 0.001 \\
\hline & Maximal & 1966 & 6.23 & 2.22 & 0.11 & \\
\hline & Medium & 2644 & 7.33 & 2.21 & 0.05 & \\
\hline
\end{tabular}

$t$ test of independent samples: $F$ 33.315, $t$ 9.6222, DF 6595, Sig 0.0001

$t$ test of independent samples: $F$ 24370, $t$ 9.088, DF 3072, Sig 0.0001

$t$ test of independent samples: $F$ 70074, DF 1, Sig 0.0001 
Table 3 Regression correlation analysis of migraine headacheassociated features

\begin{tabular}{lcr}
\hline Migraine symptom/migraine headache & $r_{12}$ & $r^{2}{ }_{12}(\%)$ \\
\hline Relief after sleep & 0.96 & 93.17 \\
Vomiting instinct & 0.94 & 89.72 \\
Vomiting & 0.90 & 81.00 \\
Photophobia & 0.84 & 71.89 \\
Nausea & 0.75 & 55.71 \\
Phonophobia & 0.75 & 55.71 \\
Vertigo & 0.23 & 5.13 \\
Other & 0.19 & 3.66 \\
Abdominal pain & 0.18 & 3.35 \\
\hline
\end{tabular}

criminant coeficient $>0.3$ ) to distinguish migraine from non-migraine headaches in children: vomiting impulse (0.945), relief after sleep (0.945), photophobia (0.523), nausea (0.379), phonophobia (0.354) and vomiting (Table 2).

Multiple analysis with multiple regression showed a very high (canonical discriminant coeficient $>0.75$ ) correlation for relief after sleep, vomiting impulse, photophobia, vomiting and photophobia with MS in children. Nausea and phonophobia have high correlation with migraine in children (0.75). Other analysed symptoms did not present a significant correlation with MS in children (Table 3).

\section{Discussion}

In the northern province of Serbia, MS appears in $8.36 \%$ $(43.95 \% \mathrm{~m}: 56.05 \% \mathrm{f})$ of children aged $3-17$. Our findings are comparable to the conclusions of other studies. Sillanpaa and Anttila found a headache prevalence of $37 \%$ in 2941 children in Finland aged 7-14 years. In $2.7 \%$ children aged 7 years and $10.6 \%$ children aged 14 years he defined migraine using Vahlquist's criteria [10]. Wöber-Bingöl et al.'s population study of migraine in children and adolescents [11] encountered migraine prevalence of $1.1 \%-5 \%$ in children and $3 \%-17.6 \%$ in adolescents. Zencir et al. [12] found overall migraine prevalence was $8.8 \%$; it was $6.7 \%$ in boys and $11.0 \%$ in girls (OR: 1.7; 95\% CI: 1.3-2.3). Balottin et al. [13] reports a mean age at onset of 4 years and 2 months, and a range of 10 months to 6 years presenting with headache symptomatology. In our group the first migraine headache attack is reported at a median age of 5.14 years (range 2.8-14 years).

Children with migraine headaches describe their attacks as identical even four times more often than children with non-migraine headaches. Migraine headache is mostly unilateral. Distinct pain location in migraine headaches is $(p<0.0001)$ connected with the age of the children and their ability to interpret it [14]. WöberBingöl et al. [15] found in 260 consecutive patients aged 3-69 that headache duration and the prevalence of unilateral, pulsating pain, photophobia and phonophobia increased in females, whereas the prevalence of aggravation by physical activity decreased with age.

Migraine headaches that affect younger children last significantly shorter: a few minutes at an average age of 4.5 years, up to $1 \mathrm{~h}$ at an average age of 5.37 years, and more than $2 \mathrm{~h}$ beyond 12.32 years. Analysing 409 children, Wöber-Bingöl et al. [16] found the duration of migraine attacks was less than $2 \mathrm{~h}$ in $19.0 \%$ of migraine patients.

Migraine headaches often occur early in the morning, with a clear prevalence in the group with rare attacks (several times a year $-78.0 \%: 22.7 \% ; p=0.0001$ ). The frequencies of migraine attacks, as well as other characteristics of childhood MS, depend on children's age. Most migraine attacks occur several times a year in children with an average age of 11.11 years and onset at the age of 5.11 years. Sleeping off the pain $(76.6 \%)$ and absence of spontaneous pain relief $(2.6 \%)$ are also among the characteristics of migraine headaches in children [14]. Senbil et al. [17] described relief of headache with sleeping or lying down in a dark, quiet room was found to be the highest specific and sensitive factor of migraine headache not included in diagnostic criteria.

In the group of children with headache who experience nausea, $83.8 \%$ have migraine and $16.1 \%$ non-migraine headaches. Among children who experience vomiting impulse, $98.7 \%$ have migraine and $1.3 \%$ have nonmigraine headaches. In the group of children who experience vomiting, $99.4 \%$ have migraine headaches and $0.6 \%$ non-migraine headaches. In the group of children with headache-associated nausea and/or vomiting instinct and/or vomiting, $83.7 \%$ have migraine headache and only $16.3 \%$ have non-migraine headaches. In the group of children who have neither nausea nor vomiting impulse, only $0.7 \%$ have migraine headaches, and $99.3 \%$ have nonmigraine headaches [14]. Our results clearly define nausea, vomiting impulse and vomiting as elements of MS attacks in children, much more frequent in younger children. Only $1.3 \%$ of children with migraine headaches at average age of 6.45 years have no nausea, vomiting impulse or vomiting. Zambrino et al. [18] report that with the exception of the presence of vomiting in migraine patients, the age at onset was not found to be a factor influencing the characteristics of the headache.

A percentage of 74.46 children at average age of 6.23 years reported recurrent abdominal pain during MS headache attacks. A percentage of 75.44 children at average age of 12.27 years had no recurrent abdominal pain during 
MS headache attacks. Among children who experience bowel movement disorders or changes in stool consistency, $97.4 \%$ have migraine and $2.6 \%$ non-migraine headaches. Considering the fact that only a small number of children have headache-associated bowel movement disorders, this symptom has a low significance, but a very high specificity [14]. Numerous authors write about abdominal migraine as one of the variants of migraine headache that typically occurred in children within the group (coded as 1.3.2 in the revised edition of IHS classification - 'Childhood periodic syndromes that are commonly precursors of migraine'). The affected children frequently develop typical migraine later [19]. Vomiting and abdominal pain are symptoms that may arise from a number of different causes. Both are noted for the absence of pathognomonic clinical features but also for the large number of other conditions to be considered in their differential diagnoses. It is important to carefully evaluate these patients, as well-being between vomiting episodes does not guarantee the absence of organic disease [20].

A percentage of 99.3 children with headache-associated phonophobia suffer from migraine headaches and only $0.7 \%$ from non-migraine headaches. Migraine-associated photophobia is reported in $89.7 \%$ of children with migraine headaches, and only in $0.2 \%$ of children with non-migraine headaches. Other vegetative symptoms (changes in skin colour, sweating, irregular breathing, precordial pain) are associated with migraine headache in $98.7 \%$ of children and in $8.8 \%$ with non-migraine headaches [14]. Migraineassociated skin pallor is found in $96.1 \%$ and dark circles under the eyes in $70.2 \%$ of children with migraine headaches $(p=0.000001)$ [14]. There is a high specificity of vegetative symptoms in migraine headaches $(97.5 \%)$ $(p=0.0001)$, which allows inclusion of various vegetative symptoms collectively into the diagnostic criteria of MS in children [14]. No age-dependent correlation was present with photophobia and phonophobia presence during MS headaches in our study group. Nausea, vomiting impulse and vomiting are basic elements of migraine attacks in children. Vegetative symptoms seem to be age dependent, with higher expression in younger children.
Highly statistically significant $(>0.3)$ discrimination factors between migraine headaches and non-migraine headaches in children, established by canonical discriminate analysis, which should be included into the definition of children MS, are as follows: relief after sleep, vomiting impulse, photophobia, nausea, phonophobia and vomiting. Wöber-Bingöl [15] found that intensity of pain, aggravation of headache by physical activity, nausea and vomiting were the most important features, and the quality of pain, photo- and phonophobia were less helpful in the differential diagnosis of migraine and tension-type headache.

Multiple analyses with multiple regressions show the strongest connection of relief after sleep, vomiting impulse, vomiting and photophobia with MS in children. Nausea and phonophobia also show slightly lower connections with migraine in children. Other analysed symptoms did not present significant correlations with childhood MS.

\section{Conclusions}

This study pointed to certain specific features of migraine headaches in children: identical signs and symptoms during individual migraine attacks, onset in early morning hours, lasting several hours and monthly migraine episode frequency, specific migraine-associated symptoms (nausea; impulse to vomit; vomiting; nausea, impulse to vomit and vomiting all together; pallor; phonophobia; photophobia; dark circles under the eyes), and cessation of symptoms after sleep. The strongest connection with MS in children had the following factors: relief after sleep, vomiting impulse, vomiting and photophobia present during MS headache. Slightly lower but still high connections are found with nausea and phonophobia. MS headache in children can be distinguished from primary non-migraine headache in children by relief after sleep, vomiting impulse, photophobia, nausea, phonophobia and vomiting.

\section{References}

1. Goadsby O, Olesen J (1996) Diagnosis and management of migraine. BMJ 312:1279-1283

2. Barlow CF (1984) Headache and migraine in childhood. Clinics in developmental medicine. No. 91 London S.I.M.P. with Blackwell Scientific, Philadelphia
3. Fontanelle LM et al (1988) Migraine in childhood: difficulties in diagnosis. Arq Neuropsiqiatr 56:553-558

4. Abu-Aref I, Macleod S (2005) Serious neurological disorders in children with chronic headache. Arch Dis Child 90:937-940
5. Battistella PA, Fiumana E, Binelli M et al (2006) Primary headaches in preschool age children: clinical study and follow-up in 163 patients. Cephalalgia 26:162-171 
6. De Diego EV, Lanteri Minet M (2005) Recognition and management of migraine in primary care: influence of functional impact measured by the headache impact test (HIT). Cephalalgia 25:184-190

7. Lipton RB, Bigal ME (2005) Migraine: epidemiology, impact, and risk factors for progression. Headache 45[Suppl 1]:S3-S13

8. Headache Classification Committee of the International Headache Society (2004) The International Classification of Headache Disorders, 2nd edition. Cephalalgia 24[Suppl 1]:9-160

9. Olesen J (2005) The International Classification of Headache Disorders, 2nd edition (ICHD-II). Rev Neurol (Paris) 16:689-691

10. Sillanpaa M, Anttila P (1983) Changes in the prevalence of migraine and other headaches during the first seven school years. Headache 23:15-19
11. Wöber-Bingöl C, Wober C, Karwautz A (1993) Diagnosis of headache in childhood and adolescence: a study of 437 patients. Cephalalgia 13:207-237

12. Zencir M, Ergin H, Sahiner $\mathrm{T}$ et al (2004) Epidemiology and symptomatology of migraine among school children: Denizli urban area in Turkey. Headache 44:780-785

13. Balottin U, Nicoli F, Pitillo G et al (2004) Migraine and tension headache in children under 6 years of age. Eur J Pain 8:307-314

14. Knezevic-Pogancev M (2003) Migrenski sindrom dece. Beograd, Todra, pp 63-74

15. Wöber-Bingöl C, Karwautz A et al (2004) Clinical features of migraine: a cross-sectional study in patients aged three to sixty-nine. Cephalalgia 24:12-17
16. Wöber-Bingöl C, Wober C, Karwautz A et al Diagnosis of headache in childhood and adolescence: a study in 437 patients. Cephalalgia 15:13-21

17. Senbil N, Gurer YK, Aydin OF, Rezaki B, Inan L (2006) Diagnostic criteria of pediatric migraine without aura. Turk J Pediatr 48:31-37

18. Zambrino CA, Balottin U, FerrariGinevra O et al (2000) Clinical characteristics of adolescent headache. Funct Neurol 15[Suppl 3]:106-115

19. d'Onofrio F, Cologno D, Buzzi MG et al (2006) Adult abdominal migraine: a new syndrome or sporadic feature of migraine headache? A case report. Eur J Neurol 13:85-88

20. Catto-Smith AG, Ranuh R (2003) Abdominal migraine and cyclical vomiting. Semin Pediatr Surg 12:254-258 